\title{
Transalpina
}

TRANSALPINA Études italiennes

$20 \mid 2017$

Edmondo De Amicis. Littérature et société

\section{Paolo Giovannini, La psichiatria di guerra. Dal fascismo alla seconda guerra mondiale}

\section{Antonella Torre}

\section{(2) OpenEdition}

12 Journals

\section{Edizione digitale}

URL: http://journals.openedition.org/transalpina/420

DOI: $10.4000 /$ transalpina.420

ISSN: 2534-5184

\section{Editore}

Presses universitaires de Caen

\section{Edizione cartacea}

Data di pubblicazione: 1 septembre 2017

Paginazione: 282-283

ISBN: 978-2-84133-857-3

ISSN: 1278-334X

Notizia bibliografica digitale

Antonella Torre, "Paolo Giovannini, La psichiatria di guerra. Dal fascismo alla seconda guerra mondiale », Transalpina [Online], 20 | 2017, online dal 19 décembre 2019, consultato il 12 novembre 2020. URL :

http://journals.openedition.org/transalpina/420 ; DOI : https://doi.org/10.4000/transalpina.420 


\section{Paolo Giovannini, La psichiatria di guerra. Dal fascismo alla seconda guerra mondiale, Milano, Edizioni Unicopli (Biblioteca di Storia contemporanea; 49), 2015, 165 p.}

In questo volume lo studioso Paolo Giovannini si concentra, in maniera del tutto originale, sulla storia della psichiatria nella seconda guerra mondiale, con ampi riferimenti agli anni del regime fascista; tematica sinora poco indagata in relazione a questo evento. Attraverso sei capitoli l'A. propone una panoramica degli studi condotti sulla storia della psichiatria di guerra, una «nuova "specialità", destinata a recitare un ruolo senz'altro di primo piano nei conflitti del XX secolo» (p. 10-11).

Gli anni Trenta sono definiti come la fase del "grande internamento » (p. 21) : la propaganda fascista predisponeva le famiglie al ricovero preventivo e incentivava l'impiego delle terapie convulsivanti (insulinoterapia, shock cardiazolico, elettroshock, ecc.), proponendole come strumenti terapeutici per chi si opponeva al regime. L'ampio e ambizioso progetto di «rinascita della stirpe» passava anche attraverso i canali dell'igiene mentale, e al grado di sufficiente validità fisica, richiesto agli italiani, doveva corrispondere il massimo livello dei requisiti di normalità psichica: «il confine fra normalità e follia apparve sempre più sfumato e incerto; le gravi ristrettezze, le paure, le angosce, i lutti, i continui timori per la sorte dei propri congiunti, amici e conoscenti, fecero sì che questa soglia non potesse essere determinata con sufficiente precisione» (p. 36).

Paolo Giovannini dedica alcune pagine alla descrizione di alcuni aspetti interessanti dal punto di vista neuropsichiatrico: gli ospedali psichiatrici, i militari, i prigionieri e la popolazione civile. La chiamata alle armi del personale sanitario provocò lo svuotamento coercitivo degli istituti di cura e assistenza: al picco di internati registrato in epoca fascista, corrispose, infatti, un notevole numero di dimissioni durante la guerra, «scaricando di fatto sulle famiglie il peso della gestione dei malati» (p. 59). Dall'analisi dello status quo della neuropsichiatria al fronte, emerge un complesso scenario di sintomatologie connesse non solo ai disturbi psichici e nervosi, ma anche ai traumi fisici subiti dai combattenti. L'A. offre al lettore uno spaccato di queste manifestazioni patologiche evidenziando le somiglianze e le peculiarità delle categorie analizzate, in relazione ai differenti contesti nei quali avevano vissuto. La stanchezza dei turni di guardia o dei lavori forzati e delle vessazioni, cui erano sottoposti i prigionieri, si arricchiva di ulteriori manifestazioni psichiatriche, quali la crescente introversione e l'insorgere di numerose manie e fobie.

$\mathrm{Al}$ termine delle ostilità, i neuropsichiatri italiani furono pressoché d'accordo nel constatare che i costi più elevati della guerra erano stati 
pagati dalla popolazione civile: "psicosi e psiconevrosi di origine bellica possedevano alcune note speciali, mentre il prolungarsi delle ostilità doveva essere considerato come un importante fattore predisponente, generando una diminuita resistenza della popolazione di fronte alle scosse emotive» (p. 145). La ben strutturata opera assistenziale, che auspicavano studiosi come Dino Origlia, non trovò un riscontro concreto, e gli effetti psicopatologici generati dal conflitto non potevano dissolversi al termine di questo. Il contributo si conclude con la menzione del XXIII Congresso nazionale della Società Italiana di Psichiatria, tenutosi a Roma nell'ottobre del 1946, al quale presero parte numerosi esperti italiani del settore. Sulla base degli Atti del Congresso, l'A. ricostruisce il dibattito di quei giorni in merito alle sorti della disciplina: nonostante le difficoltà che doveva affrontare il Paese all'indomani della guerra, tutti i partecipanti al Congresso aspiravano all'abolizione dei principi fascisti che avevano sorretto la disciplina psichiatrica e a un nuovo sviluppo della scienza, a partire da dove il progresso scientifico italiano si era arrestato.

Antonella TORRE

\section{Piccoli eroi. Libri e scrittori per ragazzi durante il ventennio fascista, Massimo Castoldi (dir.), Milan, FrancoAngeli (Storia dell'editoria), 2016, 174 p.}

Pendant les années où Mussolini fut au pouvoir, les livres et les journaux pour l'enfance furent suivis avec attention par le régime, qui comprit vite les potentialités qu'ils offraient pour inculquer aux jeunes générations une nouvelle «culture» et un nouveau guide moral, et laissa peu d'échappatoires aux écrivains et aux éditeurs désireux de faire entendre une voix différente. Cet ouvrage, qui offre une vue d'ensemble sur la littérature pour l'enfance sous le fascisme, présente les actes, recueillis par Massimo Castoldi, d'un colloque consacré aux différentes manières selon lesquelles le fascisme a agi sur la production littéraire pour la jeunesse, qui s'est tenu à Milan, à Palazzo Greppi le 5 novembre 2014.

L'une des formes du nouveau "canon littéraire» né dans l'entre-deuxguerres a été le «Bildungsroman fasciste»: un nouveau type de "roman de formation" qui donne une forme narrative à l'interprétation fasciste de l'après-guerre et de la marche sur Rome, en proposant des fictions qui retracent l'éducation politique d'un garçon, selon un schéma narratif et idéologique stéréotypé, par lequel l'histoire individuelle répète l'histoire collective (Mariella Colin). Mais plus encore que sur la littérature pour l'enfance, le fascisme a imprimé sa marque sur le livre scolaire, qui à partir 